\title{
Directional Coupler Based on Metamaterial Square CSRR Shape
}

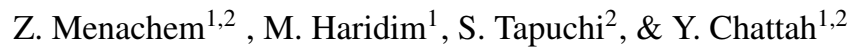 \\ ${ }^{1}$ Department of Electrical, Electronics, and Communication Engineering, Holon Institute of Technology, 52 \\ Golomb St. POB 305, Israel \\ ${ }^{2}$ Department of Electrical Engineering, Sami Shamoon College of Engineering, Israel \\ Correspondence: Z. Menachem, Department of Electrical Engineering, Sami Shamoon College of Engineering, \\ Israel. E-mail: zionm@post.tau.ac.il
}

Received: March 22, 2012 Accepted: April 6, 2012 Online Published: May 1, 2012

doi:10.5539/apr.v4n2p258 URL: http://dx.doi.org/10.5539/apr.v4n2p258

\begin{abstract}
Metamaterials are artificial structures that can be designed to exhibit specific electromagnetic properties that are not in the nature. In this paper, we design a directional coupler, based on the theory of the split-ring resonators (SRRs), and the complementary SRR (CSRRs). The experiments and simulations of the directional coupler are based on the theory of the square structure (and not based on the circular structure) of the SRR and CSRR. The advantage of this circuit is that the area of the coupling is great as regards to the coupler based on the circular structure. The results of simulation and measurement with the miniature structures show the backward-wave phenomenon of the left-handed (LH) material.
\end{abstract}

Keywords: directional coupler, metamaterial square CSRR shape, backward waves

\section{Introduction}

In the recent years, artificial metamaterials have been a subject of growing interest, also in the microwave and antenna regimes. Metamaterials are artificial structures that can be designed to exhibit specific electromagnetic properties that are not in the nature. Metamaterials with negative permittivity and permeability referred to lefthanded (LH) materials, have received substantial attention in scientific and engineering communities. Veselago explained the possible existence of LH materials and anticipated their unique electromagnetic properties, and showed that LH materials support electromagnetic waves with group and phase velocities that are antiparallel, known as backward wave, a phenomenon which is associated with negative index of refraction (Veselago, 1968). According to Veselago, the negative valued permeabilities enable us to realize left-handed metamaterials.

Pendry et al. created in 1999 the theory of the LH metamaterial (LHM) (Pendry et al., 1999). The idea was to create materials with negative permeability. They show how to design structures made from nomagnetic thin sheets of metal, which respond to microwave radiation as if they used with an effective magnetic permeability. A wide range of permeabilties can be achieved by varying the parameters of the structures. Split ring resonator (SRR) is a metamaterial structure that is used in microwave and antenna applications. The SRR pattern is printed on a dielectric substrate in the form of conducting strip lines made of good conductors such as copper or gold. An SRR array can provide highly positive or highly negative effective permeability values over some definite frequency bands (Pendry et al., 1999). An SRR based unit cell and SRR based array structures with various sizes have been designed and fabricated in order to investigate the effects of array sizes on the transmission characteristics (Ekmekci et al., 2009). Transmission characteristics were obtained by both simulations and experiments. The results have shown that increasing array size increases the depth of the transmission minimum at the resonance frequency and also widens the stop-bandwidth. The resonance frequency of the array is shifted either towards the lower frequencies or towards the higher frequencies depending upon the array dimension at which the number of array elements is increased.

Directional couplers have four ports. The first port is regarded as the input, the second port is regarded as through port, the third port is regarded as the coupler port, and the firth port is regarded as isolated port. One port of the directional coupler is isolated from the input port. Structures in coupled-line couplers were proposed (Liu et al., 2002), where a symmetric LH enhanced forward coupler was demonstrated. The forward coupling phenomena between artificial LH transmission lines have been explored on the basis of a transmission line approach of LH 
materials. A LH forward coupler in the form of an ideal lumped-element network was presented (Liu et al., 2002), in order to exhibit strong forward coupling that increases with decreasing of frequency. It was demonstrated that this coupler requires a shorter coupling length in comparison with the conventional right-handed coupled-line forward couplers, and hence makes the device much more compact. A novel broadband LH coupled-line backward coupler with arbitrary coupling level was demonstrated by Caloz, et al. (2003). This coupler is composed of two LH transmission lines (TL) constituted of series interdigital capacitors and shunt shorted-stub inductors. The coupler was shown to exhibit a broad bandwidth. Parametric results illustrate the versatility of the LH coupler and its strongly enhanced backward coupling compared with the conventional coupled-line coupler. A novel backward directional coupler, composed of a conventional microstrip line and composite right/left-handed (CRLH) line, was proposed by Caloz, et al. (Caloz et al., 2004). This coupler was functionally backward but it is based on a forward-type coupling phenomenon.

The split-ring resonator defected ground structure is applied to the wideband filter design (Hou et al., 2008). A micro-strip band-pass filter with a transmission zero at right out-of-band are designed using the equivalent-circuit analysis and curve-fitting method, which is then realized in the actual compact structure, making use of lumped chip capacitors and T-shaped open-circuit stub to achieve series and shunt capacitance, respectively.

A novel backward-wave coupler consisting of the combination of a conventional microstrip line and a left-handed microstrip line has been presented (Jarauta et al., 2006). Contra-directional power flow in the coupler has been indicative of left-handedness in the backward transmission line, which has been artificially fabricated by means of complementary SRRs (CSRRs), etched in the ground plane, and an array of T-shaped capacitive elements, etched in the conductor strip. The coupler provided more than $20-\mathrm{dB}$ improvement in the coupling level in comparison with the traditional $\lambda / 4$ coupler with similar dimensions in the coupling region.

The objective in this paper is to design coupler based on the theory of the SRR and CSRR in the case of the miniature structures in order to understand about the backward-wave phenomenon of the LH material. The experiments and simulations of the directional coupler are based on the theory of the square structure of the SRR and CSRR. The backward-wave phenomenon of the LH material is very interesting.

\section{Coupler Design and the Results}

A square shaped CSRRs topology and the relevant dimensions are shown in Figure 1(a), where the relevant parameters of the CSRR element wire are $1=6.28 \mathrm{~mm}, \mathrm{~g}=0.5 \mathrm{~mm}$, and $\mathrm{w}=0.4 \mathrm{~mm}$. The distance between the periodic elements is $1.72 \mathrm{~mm}$. Figure 1(b) represents the periodic structure that consists of three square shaped SRR structure. The objective in this paper is to design coupler based on the theory of the SRR and CSRR in order to understand the backward-wave phenomenon of the LH material. Backward-wave phenomenon means that the propagation of the wave is in the opposite direction as regards to the conventional case. Directional coupler is passive device with four ports, as shown in Figure 1(b). A coupler has four ports, input, transmitted, coupler, and isolated. According to Figure 1(b), for the conventional case, port 1 is the incident port, port 2 is the transmitted port, port 3 is the isolated port, and port 4 is the coupled port. For the metamaterial coupler, port 4 is the isolated port, and port 3 is the coupled port, as shown in Figure 1(b). Thus, port 3 in the metamaterial coupler is coupled port instead of port 4 in the conventional coupler. The implementation of a simple coupler is based on the coupling of two transmission lines. This is known as a coupled line coupler.

There are two traditional topologies of implementation the coupler, backward (port 3 is coupled port and port 4 is isolated port), and forward (port 3 is isolated port and port 4 is coupled port), as shown in Figure 1(b). The coupler can be designed by using the $S$-matrix with four ports network. For a backward coupler, the requirement for S-matrix is given by Marques, et al., (2007) as follows

$$
\begin{aligned}
& S_{11}=0 \quad, \quad S_{21}=\frac{\sqrt{1-k^{2}}}{\sqrt{1-k^{2}} \cos \phi+j \sin \phi}, \\
& S_{31}=\frac{j k \sin \phi \quad}{\sqrt{1-k^{2}} \cos \phi+j \sin \phi} \quad, \quad S_{41}=0,
\end{aligned}
$$

where $\phi$ is the phase shift, $\mathrm{k}$ is the coupling factor that is given by $k=\left(Z_{o e}-Z_{o o}\right) /\left(Z_{o e}+Z_{o o}\right), Z_{o e}$ and $Z_{o o}$ are the even characteristic impedance and the odd characteristic impedance, respectively. According to the assumption of a symmetric structure, even and odd mode analysis lead to the coefficients of the above S-matrix, and the condition to obtain perfect matching at the input port is given by $Z_{o}=\sqrt{Z_{o e} Z_{o o}}$. The assumption is that the phase 
shift $\phi$ (or the phase velocity) for the even and odd modes is roughly the same (which is in turn an assumption valid for quasi-TEM wave propagation). Coupling (i.e., $S_{31}$ depends on the electrical length of the coupled lines and on the coupling factor k). The optimum coupling is obtained for $\phi=\pi / 2$. In this case, the coupling is $C=\left|S_{31}\right|=\mathrm{k}$. Thus, in these backward wave couplers, maximum coupling depends on the difference between the characteristic impedance for the even and the odd modes. In practice, the coupling levels achievable with these coupler are very limited. Thus, the coupling is defined by the even and the odd characteristic impedance. It is difficult to implement a good coupling of the backward coupler, because it is limited by the geometrical limitations. On the other hand, it is easy to get this feature of the coupling by using the metamaterial implementation. In metamaterial, the couplers are relatively small, depending on the required coupling, and the bandwidth is reasonably good.

In order to produce the band pass filter we had a capacitor in the microstrip line and then the equivalent circuit for the new CSRR, as shown in Figure 2. The production of the band pass filter is based on the equivalent circuit for the CSRR, as explained by Hou et al. (2008).

The design of our coupler is based on the article of Jarauta, et al. (2006), but the experiments and simulations of the directional coupler are based on the theory of the square structure (and not based on the circular structure) of the SRR and CSRR. The advantage of this circuit is that the area of the coupling is great as regards to the coupler based on the circular structure. Figures 3(a) and 3(b) show the backward coupler composed of a conventional microstrip line and an LHM line with three metamaterial cells. The experimental results are given by CST Microwave Studio program as simulation and Agilent E5071C Network Analyzer. The coupling of S31 is 2.4 $\mathrm{GHz}$ for $36 \mathrm{~mm}$ given length, as shown in Figure 4. From the simulation we can see that the coupler is working in the wireless local area network (WLAN) bandwidth. The transmission $S_{21}$ is around $-3 \mathrm{~dB}$ in the coupling frequencies and get less then $-6 \mathrm{~dB}$ in the $S_{31}$ (coupling port). This is an asymmetric coupler. The comparison between the simulation and the measured coupler is shown in Figure 4. This result agrees with the experimental and simulation results.

\section{Conclusions}

In this study, we designed a directional coupler, based on the theory of the split-ring resonators (SRRs), and the complementary SRR (CSRRs). A square shaped CSRRs topology and the relevant dimensions are shown in Figure 1(a), where the relevant parameters of the CSRR element wire are $1=6.28 \mathrm{~mm}, \mathrm{~g}=0.5 \mathrm{~mm}$, and $\mathrm{w}=0.4$ $\mathrm{mm}$. The distance between the periodic elements is $1.72 \mathrm{~mm}$. The periodic structure that consists of three square shaped CSRR structure is shown in Figure 1(b). The advantage of this circuit is that the area of the coupling is great as regards to the coupler based on the circular structure. The experiment and simulations of the directional coupler are based on the theory of the square structure (and not based on the circular structure) of the SRR and CSRR. The results of simulation and measurement with the miniature structures show the backward-wave phenomenon of the LH material. Backward-wave phenomenon means that the propagation of the wave is in the opposite direction as regards to the conventional case.

Directional coupler is passive device with four ports, as shown in Figure 1(b). A coupler has four ports, input, transmitted, coupler, and isolated. According to Figure 1(b), for the conventional case, port 1 is the incident port, port 2 is the transmitted port, port 3 is the isolated port, and port 4 is the coupled port. For the metamaterial coupler, port 4 is the isolated port, and port 3 is the coupled port, as shown in Figure 1(b). Thus, port 3 in the metamaterial coupler is coupled port instead of port 4 in the conventional coupler. The implementation of a simple coupler is based on the coupling of two transmission lines. This is known as a coupled line coupler. Namely, there are two traditional topologies of implementation the coupler, backward (port 3 is coupled port and port 4 is isolated port), and forward (port 3 is isolated port and port 4 is coupled port), as shown in Figure 1(b). The coupler can be designed by using the S-matrix with four ports network. Coupling (i.e., $S_{31}$ depends on the electrical length of the coupled lines and on the coupling factor $\mathrm{k}$ ). The optimum coupling is obtained for $\phi=$ $\pi / 2$, where $\phi$ is the phase shift. In this case, the coupling is $C=\left|S_{31}\right|=\mathrm{k}$, where k is the coupling factor that is given by $k=\left(Z_{o e}-Z_{o o}\right) /\left(Z_{o e}+Z_{o o}\right)$.

In the backward wave couplers, maximum coupling depends on the difference between the characteristic impedance for the even and the odd modes. In practice, the coupling levels achievable with these coupler are very limited. Thus, the coupling is defined by the even and the odd characteristic impedance. It is difficult to implement a good coupling of the backward coupler, because it is limited by the geometrical limitations. On the other hand, it is easy to get this feature of the coupling by using the metamaterial implementation. In metamaterial, the couplers are relatively small, depending on the required coupling, and the bandwidth is reasonably good. 
In order to produce the band pass filter we had a capacitor in the microstrip line and then the equivalent circuit for the new CSRR, as shown in Figure 2. Figures 3(a) and 3(b) show the backward coupler composed of a conventional microstrip line and an LHM line with three metamaterial cells. The experimental results are given by CST Microwave Studio program as simulation and Agilent E5071C Network Analyzer. The coupling of S31 is $2.4 \mathrm{GHz}$ for $36 \mathrm{~mm}$ given length, as shown in Figure 4. From the simulation we can see that the coupler is working in the WLAN bandwidth. The transmission $S_{21}$ is around $-3 \mathrm{~dB}$ in the coupling frequencies and get less then $-6 \mathrm{~dB}$ in the $S_{31}$ (coupling port). This is an asymmetric coupler. The comparison between the simulation and the measured coupler is shown in Figure 4. This result agrees with the experimental and simulation results.

\section{References}

Caloz, C., Sanada, A., Liu, L., \& Itoh, T. (2003). A broadband left-handed (LH) coupled-line backward coupler with arbitrary coupling level, IEEE-MTT Int. Symp., 1, 317-320.

Caloz, C., \& Tatsuo Itoh, T. (2004). A novel mixed conventional microstrip and composite Right/Left-Handed backward-wave directional coupler with broadband and tight coupling characteristics, IEEE Microwave and Wireless Components Letters, 14, 31-33. http://dx.doi.org/10.1109/LMWC.2003.821506

Ekmekci, E., Topalli, K., Akin, T., \& Turhan-Sayan, G. (2009). Effects of array dimensions on the resonance characteristics of SRR type metamaterial arrays with small sizes: simulations and experiments, Progress In Electromagnetics Research Symposium Proceedings, 83-86.

Hou, Z.Z., Li, X.X., \& Hao, C.K. (2008). Design of wideband filter using split-ring resonator DGS, Progress In Electromagnetics Research Symposium, Hangzhou, China, 33-36.

Jarauta, E., Laso, M. A. G., Lopetegi, T., Falcone, F., Beruete, M., Baena, J. D., Marcotegui, A., Bonache, J., Garcia, J., Marques, R., \& Martin, F. (2006). Novel microstrip backward coupler with metamaterial cells for fully planar fabrication techniques, Microwave and Optical Technology Letters, 48, 1205-1209. http://dx.doi.org/10.1002/mop.21579

Liu, L., Caloz, C., Chang, C., \& Itoh, T. (2002). Forward coupling phenomena between artificial left-handed transmission lines, J. Appl. Phys., 92, 5560-5565. http://dx.doi.org/10.1063/1.1512682

Marques, R., Martin, F., \& Sorolla, M. (2007). Metamaterials with negative parameters. Wiley, $246-252$.

Pendry, J. B., Holden, A. J., Robbins, D. J., \& Stewart, W. J. (1999). Magnetism from conductors and enhanced nonlinear phenomena, IEEE Trans. Microwave Theory Tech., 47, 2075-2084.

Veselago, V. G. (1968). The electrodynamics of substances with simultaneously negative values of $\epsilon$ and $\mu$, Soviet Phys. Uspekhi, 10, 509-514. http://dx.doi.org/10.1070/PU1968v010n04ABEH003699 


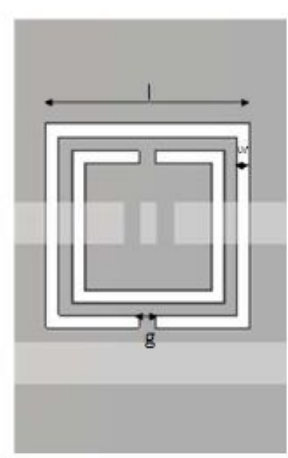

(a)

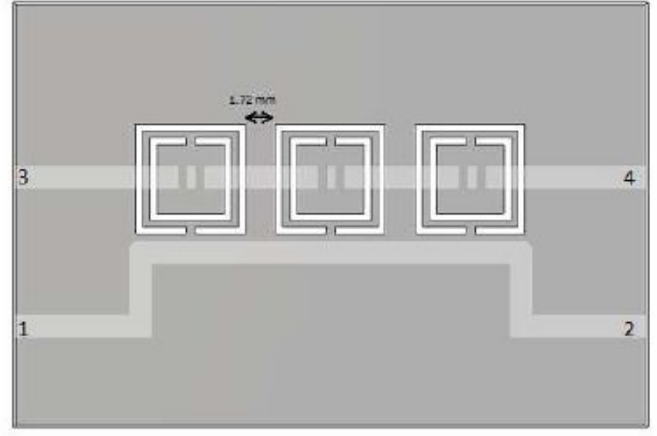

(b)

Figure 1. (a) Square shaped CSRRs topology and relevant dimensions for CSRR unit cell. (b) The simulated model of the periodic structure that consists of three square shaped CSRR structure

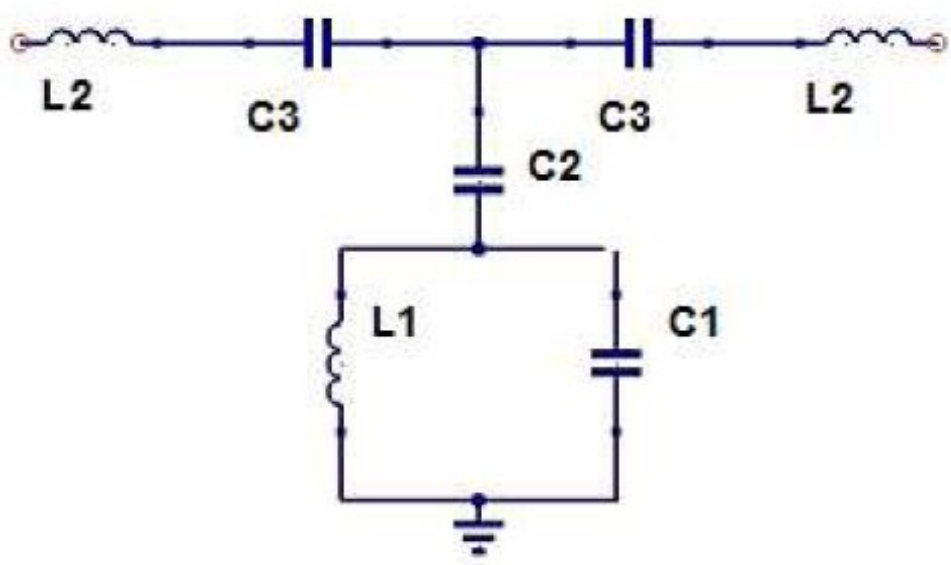

Figure 2. Equivalent circuit of CSRR unit cell with a capacitor on the microstrip line

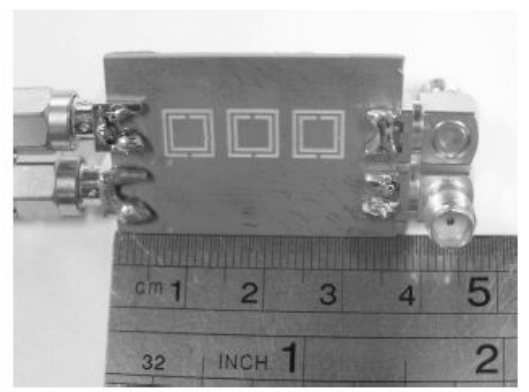

(a)

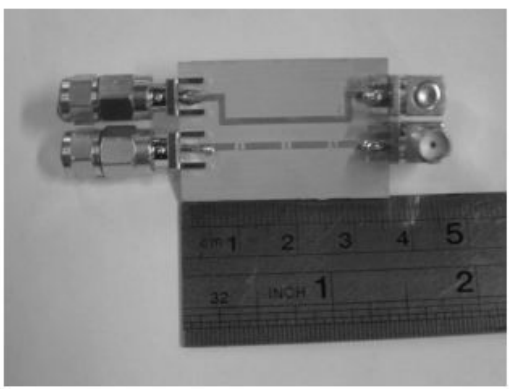

(b)

Figure 3. Backward coupler composed of a conventional microstrip line and LHM line with three metamaterial cells 


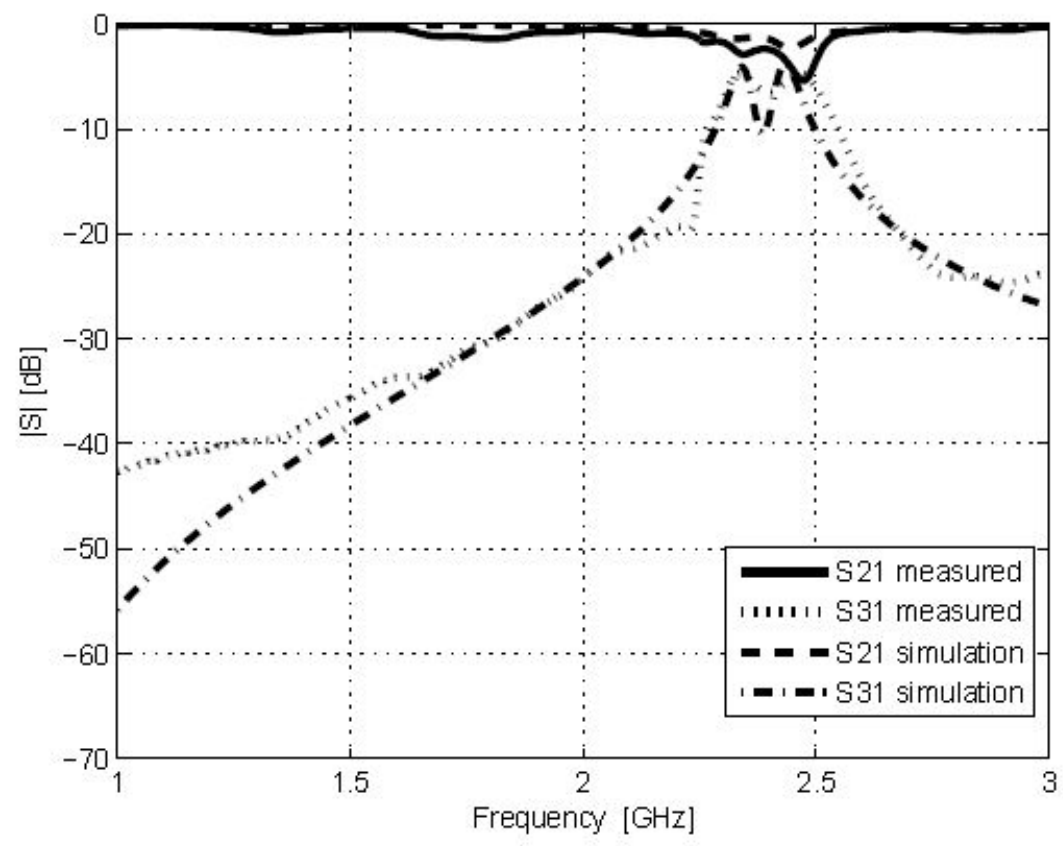

Figure 4. Comparison between the simulated S-parameters and the measured coupler for three-cell backward coupler 